\title{
Infection and Microparticles may Cause Complication of Atherosclerotic Plaques
}

Renata Nishiyama Ikegami ${ }^{1}$, Joyce Tiyeko Kawakami ${ }^{1}$, Dulcineia Saes Parra Abdalla ${ }^{2}$, Raul Dias Santos ${ }^{1}$, Roberto Kalil Filho ${ }^{1}$, Jose Antônio Franchini Ramires ${ }^{1}$ and Maria de Lourdes Higuchi ${ }^{1 *}$

${ }^{1}$ Heart Institute (InCor) of Clinical Hospital, Medical School, University of São Paulo, São Paulo, Brazil

${ }^{2}$ Faculty of Pharmaceutical Sciences, University of São Paulo, São Paulo, SP, Brazil

\begin{abstract}
Atherosclerosis is frequently associated with diabetes, obesity, metabolic syndrome and oxidized low density lipoproteins (oxLDL). Many studies have reported association of infection with such disorders, but with controversial results. In our view, these findings have relationship with technical differences. We showed previously, presence of infectious agents inside of ruptured plaque, using immunohistochemistry and electron microscopy. More recently, we detected Electron Lucent microparticles (ELMP) and Mycoplasma pneumoniae (Mp) lipoproteins in vulnerable plaques (VP).
\end{abstract}

However, we have interest to know if ELMPs contain Mp lipoprotein antigens and if they are related to oxLDL and plaque vulnerability.

Methods: We studied three groups of coronary arteries: VP (vulnerable plaque; $n=13$ ), stable plaques (SP; $n=7$ ), and normal arteries (NA; $n=7)$. All cases were studied by immuno electron microscopy, and the mean numbers of ELMPs, oxLDL and Mp antigens, inside and outside ELMP, were obtained. Double colloidal immunogold particles (anti-oxLDL and anti-Mp) allowed the simultaneous localization of both antigens.

Results: There was a significant higher amount of ELMPs in VP, with positive dots for both oxLDL and Mp antigens inside them, compared to other two groups $(p<0.01)$. Mp and oxLDL antigens were co-localized in lipidic nanoparticles intra ELMPs, showing positive correlation ( $r=0.60 ; P=0.04)$. High amount of oxLDL and Mp antigens extra ELMPs were seen in VP, but not in stable plaques.

Conclusion: Plaque vulnerability in atherosclerosis may be related to presence of ELMPs, containing $M$. pneumoniae lipoproteins and oxLDL. We hypothesized that $M$. pneumoniae lipoproteins oxidation could be a mechanism for this association. However, further data are necessary to prove this hypothesis.

Keywords: Atherosclerosis; Microparticle; Infection; Vulnerable plaque

\section{Introduction}

Atherosclerosis is a chronic inflammatory disorder involving the intima and adventitia layers [1], but with unclear mechanism for development of such inflammation. It is frequently associated with oxidized low density lipoproteins (oxLDL), secondary to dyslipidemia, diabetes, obesity, hypertension or metabolic syndrome. Some studies have reported association of gut microbiota with such disorders, but with controversial results [2,3]. The lack of standardization and technical problems in DNA extraction is apparently an important part of these discrepancies [4]. Other works have demonstrated that infection by bacteria and/or viruses may contribute on the pathogenesis of atherosclerosis either via direct infection of vascular cells or via the indirect effects of cytokines or acute phase proteins induced by infection [5]. In previous papers, comparing ruptured thrombosed plaques with equistenotic stable plaques from autopsies of patients died due to acute myocardial infarction, we described great amount of Mycoplasma pneumoniae (Mp) in association with Chlamydophila pneumoniae, in ruptured plaques. We used different techniques (immunohistochemistry, in situ hybridization and electron microscopy), to prevent errors in diagnosis of such microbes [6]. After that, we studied in vivo vulnerable plaques (VP), which were removed by atherotomy. VP are known to be larger, with increased amount of lipid in the plaque, and thin fibrous cap [7]. This study showed, by electron microscopy, Microparticles (MPs) with electron lucent (EL) content, in close association with Mp bodies, mainly in myxoid matrix foci [8].
Mycoplasmas are the smallest auto-replicating microorganisms, having unique characteristics, such as absence of an outer wall and need of cholesterol for survival. They induce alterations in the immunologic system of the host and, therefore, may favor the proliferation of other infectious agents [6].

Oxidative burst as an innate immune response to infection, aiming to neutralize microbial effects, may promote the appearance of oxidized lipids inducing inflammatory response $[9,10]$. Whilst these changes may be beneficial from the perspective of host defense, if becoming chronic, they likely increase the risk of atherosclerosis. In particular, oxidation of lipoproteins, resulting from an imbalance of the proand antioxidant equilibrium, is involved in the pathologic process of atherosclerosis, changing cellular functions [11].

Microparticles (MP) are vesicles larger than $100 \mathrm{~nm}$ diameters,

*Corresponding author: Maria de Lourdes Higuchi, Cardiac Pathology Laboratory of Heart Institute of Clinical Hospital, Medical School, University of São Paulo, Eneas de Carvalho Aguiar, 44 (-1 BI.II), CEP: 05403-000 São Paulo-SP, Brazil, Tel: +55 11-26615541; E-mail: anplourdes@incor.usp.br

Received February 26, 2014; Accepted April 20, 2015; Published April 28, 2015

Citation: Ikegami RN, Kawakami JT, Abdalla DSP, Santos RD, Filho RK, et al (2015) Infection and Microparticles may Cause Complication of Atherosclerotic Plaques. J Diabetes Metab 6: 537. doi:10.4172/2155-6156.1000537

Copyright: @ 2015 lkegami RN, et al. This is an open-access article distributed under the terms of the Creative Commons Attribution License, which permits unrestricted use, distribution, and reproduction in any medium, provided the original author and source are credited. 
derived from the plasma membrane of endothelial cells, macrophages, platelets or smooth muscle cells, but also from microbes. MPs are believed to be involved in oxidative processes and inflammation may have a role in development of plaque vulnerability and/or thrombosis [12]. MP associated with tissue factor activity is elevated in the blood in cancer patients associated with thrombosis [13]. Indeed, circulating and platelet-derived MPs enhance thrombosis on atherosclerotic plaques [14]. Effective lipid-lowering treatment may prevent the development of premature cardiovascular disease by reducing LDL and vascularinflammatory cell activation and inducing MPs reduction [15].

In the present, our objective was to analyze if the Electron lucent MPs were increased in vulnerable plaques, in association with oxLDL in correlation with Mp lipoproteins.

\section{Material and Methods}

Material for this research was collected at Heart Institute (InCor) of Clinical Hospital of Medical School from University of São Paulo, after approval by the Ethical and Scientific Committee.

Coronary plaques from three groups were studied:

-Vulnerable plaques (VP) -were obtained during interventional pre-stenting Directional Coronary Atherectomy, in 13 patients (pts.) ( 10 men and 3 women, mean age 66.5 years), diagnosed as unstable angina, defined as recent onset of chest pain and eletrocardiographic changes (ST-T) without elevation of cardiac necrosis markers. Culprit plaque was defined by the invasive cardiologist as subocclusive disease of artery lumen, reduced TIM coronary flux, correspondent to regional electrocardiographic changes. No pts. of this group had any infection or had used antibiotics.

- Coronary with stable plaques (SP) was obtained from 9 pts. ( 8 men and 1 woman, mean age 49 years). All of these patients had ischemic cardiomyopathy and were submitted to heart transplantation. Coronary arteries were studied by dissection immediately after removal of heart. Then, the arteries were transversally and serially sectioned, to identify the stable plaques by fibrotic macroscopic aspect and microscopy exhibiting thick fibrous cap and low lipid content [16,7].

-Normal coronary arteries (NA) were obtained from heart transplant receptors, which were collected immediately after removal of the heart. We had arteries from 7 pts. without atherosclerosis ( 5 men and 2 women, mean age 42,5 years), with diagnosis of severe chagasic or idiopathic cardiomyopathy, and with no obstruction of coronary artery by microscopic study. The patients did not have alteration in the lipid levels.

\section{Rapid inclusion method for transmission electron microscopy}

The inclusion was performed following the procedure described by Duarte et al. [17], with modifications summarized below. The fragments were fixed in $3 \%$ glutaraldehyde and post-fixed in $1 \%$ osmium tetroxide. Then, were washed in saline solution and incubated in $0.5 \%$ uranyl acetate. The sections were dehydrated in $70 \%$ ethanol and 2.2-dimethoxypropane acidified, followed by fixation in acetone and $4 \%$ copper sulphate. Inclusion was achieved with a mixture containing resin EPON Resin 812 polybed $F$ araldite (1:1) and polymerization in $100^{\circ} \mathrm{C}$, for 1 hour. Blocks were cut with an ultra-microtome to a thickness of $60-70 \mathrm{~nm}$ and placed on 200 mesh electron microscopy grid coated with Parlódio film.

\section{Double immunogold labelling in electron microscopy}

In order to remove Araldite resin, to expose the antigenic sites, grids were incubated in sodium metaperiodate solution of $0.5 \mathrm{M}$ for 15 minutes at room temperature (RT). Ultrathin sections were washed with distilled water and incubated with Protein Block Serum free (Dako Cytomation, California, USA/ Ref X0909) for 30 minutes at $\mathrm{RT}$, and then incubated with primary rabbit polyclonal Mp antibody (Fitzgerald, MA, USA, Cat\# 20MR54) 1:100 diluted for 20 hours at $4^{\circ} \mathrm{C}$ in a humidified chamber. They were washed in PBS and incubated for 1 hour at RT with secondary antibody (anti-rabbit IgG, 1:20 diluted) gold conjugated, $15 \mathrm{~nm}$ (Sigma Immunochemical, Saint Louis, MO, USA). The grids were washed in PBS and rinsed in distilled water. On the same grid, similar procedure was done with mouse monoclonal oxLDL antibody (clone 2C7OSF10, donated by Professor Dulcineia Abdalla, School of Pharmaceutical Sciences of University of São Paulo, Sao Paulo, Brazil), 1:20 diluted for 20 hours at $4^{\circ} \mathrm{C}$ in a humidified chamber. Then, were incubated with secondary antibody (anti-mouse IgG, 1:20 diluted) gold conjugated, $5 \mathrm{~nm}$ (Sigma Immunochemical, Saint Louis, MO, USA). The grids were washed in PBS, rinsed in distilled water and then counterstained with $5 \%$ lead citrate.

\section{Analysis}

At electron microscopy we measured the diameter of ELMPs and counted their mean numbers. By immunohistochemistry, double colloidal immunogold particles ( $5 \mathrm{~nm}$ for anti-oxLDL and $15 \mathrm{~nm}$ for anti-Mp) allowed the simultaneous localization of both antigens and counting the mean number of positive dots $/ \mu \mathrm{m}^{2}$ inside and outside ELMP, in 7 photos 50.000X magnification of each case.

Statistical Analysis: Student $t$ test was used to detect differences between VP group from SP, and Kruskal Wallis to compare both group with NA. A correlation of Pearson's test was used to find a relationship between oxLDL and Mp antigens. Statistical significance was considered when $\mathrm{p}<0.05$.

\section{Results}

VP group had increased numbers of ELMPs than NA and SP groups. Table 1 shows the mean numbers of ELMPs and their contents in the three different groups of coronary arteries. We observed higher number of positive dots for Mp and oxLDL antigens, inside ELMPs of VP group than in ELMPs of the other two groups (NA and SP). Double immunogold labeling in electron microscopy showed that $\mathrm{Mp}$ and oxLDL antigens co-localized inside ELMPs (Figure 1), the quantity of both antigens are correlated $\mathrm{r}=0.60, P=0.04$. Higher mean numbers of oxLDL antigens outside ELMPs were seen in VP, but not in SP and NA respectively $(6.96 \pm 3.72,4.34 \pm 4.63,3.21 \pm 2.07$, respectively). The same occurred regarding Mp antigens outside ELMPs (3.20 $\pm 3.13,0.19$ $\pm 0.18,0.91 \pm 1.05$, respectively). ELMPs of VP and/or SP have smaller

\begin{tabular}{|l|c|c|c|}
\hline & \multicolumn{3}{|c|}{ Electron Lucent Microparticles } \\
\hline Variables & $\begin{array}{c}\text { Mean } \\
\text { numbers(SD) }\end{array}$ & $\begin{array}{c}\text { Mycoplasmapneumoniae } \\
\text { oxLDL }\end{array}$ & Inside \\
\hline Vulnerable Plaques & $2.01(1.14)$ & $0.85(0.74)$ & $1.77(1.36)$ \\
\hline Stable Plaques & $0.81(0.60)$ & $0.00(0.00)$ & $0.31(0.26)$ \\
\hline Normal Arteries & $0.67(0.50)$ & $0.23(0.42)$ & $0.21(0.17)$ \\
\hline VP vs SP * & 0.02 & 0.008 & 0.01 \\
\hline VP vs NA* & 0.009 & 0.06 & 0.008 \\
\hline SP vs NA * & 0.64 & 0.17 & 0.39 \\
\hline
\end{tabular}

*(P of $t$ test)

Table 1: Mean numbers $/ \mu m^{2}$ of Electron lucent microparticles (ELMPs). Mycoplasma pneumoniae (Mp) and oxLDLantigens inside ELMPs from vulnerable plaques (VP), stable plaques (SP) and normal arteries (NA). 
Citation: Ikegami RN, Kawakami JT, Abdalla DSP, Santos RD, Filho RK, et al. (2015) Infection and Microparticles may Cause Complication of Atherosclerotic Plaques. J Diabetes Metab 6: 537. doi:10.4172/2155-6156.1000537

Page 3 of 4

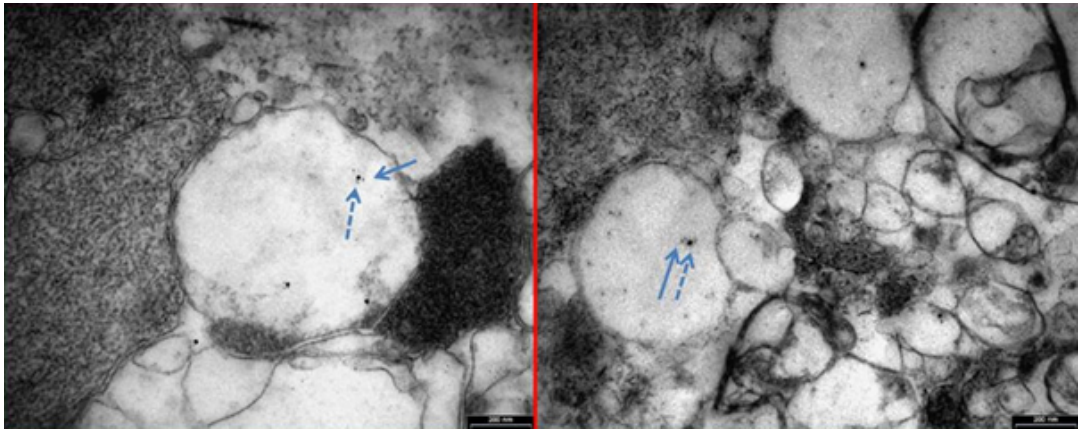

Figure 1: Immunoeletron microscopy of coronary vulnerable plaques exhibiting electron lucent microparticles (ELMPs) containing double immuno stained lipidicnanoparticles: oxLDL - thin arrow (5nm coloidal gold) and Mp- large interrupted arrow (15 nm coloidal gold) antigens.

diameters than in NA $(0.04 \pm 0.02,0.07 \pm 0.02,0.25 \pm 0.05$, respectively). All cases of all groups presented at least one ELMP, however in normal, almost did not have M. pneumoniae content.

\section{Discussion}

The chronic atherosclerotic inflammatory process on the artery wall segments is usually related to oxLDL, free radicals and endothelial dysfunction $[18,19]$. However, cholesterol appears to be a secondary factor and not a cause of atherosclerosis. Some contradictory findings have shown that high cholesterol is not a risk factor for coronary heart disease, in women and old individuals [20]. Immune complex and its aggregation may also be enhanced by autoantibodies against thiolated LDL and oxLDL. On the other hand, oxLDL, free radicals and endothelial dysfunction are characteristics associated to the presence of microparticles in human atheromas [22-24]. MPs are elevated in the blood of cancer pts and were associated to thrombosis [25]. MPs are enhanced with thrombosis in atherosclerotic plaques [14].

Infection, microparticles and oxLDL have frequently been related to development of vessel inflammation, atherosclerosis and plaque vulnerability, inducing instability and activation of endothelium.

We have described in vulnerable plaques electron lucent microparticles (ELMPs), which had characteristics of archaea, and Mycoplasma pneumoniae antigens [26,13]. Now, we looked for an interaction between ELMPs, Mp antigens and oxLDL which could be an explanation for the inflammation and thrombosis in ruptured plaques.

We found increased numbers of all these three elements in VP group when compared to SP and NA groups, and a strong correlation between oxLDL and M.pneumoniae antigens, inside ELMPs, in VPs. The quantity of both antigens are correlated $(r=0.60, P=0.04)$, and we do not know the clinical meaning. However, increased levels of M.pneumoniae in patients after AMI were described [21]. The VP group corresponded to older atherosclerotic patients, but no other risk factors such as use of statin and smoking habit were different among the stable vs. unstable group. We hypothesized that ELMPs internalize lipoproteins from M.pneumoniae, oxidizing them. These complexes are seen in large amount externally to the microparticles, and are also present in the serum [6].

This is in accordance with other authors, who proposed that lipoproteins would constitute an innate immune system response by binding and inactivating microorganisms and their toxic products, through formation of circulating complexes. In the case of a massive microbial invasion, these complexes may aggregate, in particular in the presence of hyperhomocysteinemia, because homocysteine thiolactone causes aggregation and precipitation of thiolated LDL.

Homocysteine activates cytokines and pro-inflammatory molecules, such as C-reactive protein (CRP) and up-regulates reactive oxygen species. CRP binds oxidized LDL and oxidized phospholipids, enhancing phagocytosis to form foam cells. Obstruction of the vasavasorum by aggregated lipoprotein complexes may result in local ischemia in the arterial wall, intramural cell death, bursting of the capillary, and escape of microorganisms into the intima, all of which lead to inflammation and creation of vulnerable plaques [20].

On the other hand, a symbiotic life between archaeal MPs with mycoplasma may lead to proliferation of mycoplasma and archaea, both are capable to oxidize entrapped lipoproteins increasing virulence of co-infected microbes [8].

\section{Conclusion}

Plaque vulnerability in atherosclerosis may be related to presence of ELMPs, containing co-localized M. pneumoniae lipoproteins and oxLDL. We hypothesized that M. pneumoniae lipoproteins oxidation could be a mechanism for this association. However, further data are necessary to prove this hypothesis.

\section{Acknowledgement}

This study was partially supported by CNPq (ConselhoNacional de Pesquisa) and Fundação Zerbini.

\section{References}

1. Rosenfeld ME (2013) Inflammation and atherosclerosis: direct versus indirect mechanisms. Curr Opin Pharmacol 13: 154-160.

2. Karlsson F, Tremaroli V, Nielsen J, Bäckhed F (2013) Assessing the human gut microbiota in metabolic diseases. Diabetes 62: 3341-3349.

3. Hanage WP (2014) Microbiology: Microbiome science needs a healthy dose of scepticism. Nature 512: 247-248.

4. Pekkala S, Munukka E, Rintala A, Houvinen P (2014) The microbiome studies in metabolic diseases have advanced but are poorly standardized and lack a mechanistic perspective. J Diabetes Metab 6: 480.

5. Rosenfeld ME, Campbell LA (2011) Pathogens and atherosclerosis: update on the potential contribution of multiple infectious organisms to the pathogenesis of atherosclerosis. Thromb Haemost 106: 858-867.

6. Higuchi Mde L, Reis MM, Sambiase NV, Palomino SA, Castelli JB, et al. (2003) Coinfection with Mycoplasma pneumoniae and Chlamydia pneumoniae in ruptured plaques associated with acute myocardial infarction. Arq Bras Cardiol 81: 12-22, 1-11.

7. Bezerra HG, Higuchi ML, Gutierrez PS, Palomino S, Silvestre JM, et al (2001) Atheromas that cause fatal thrombosis are usually large and frequently accompanied by vessel enlargement. Cardiovascular Pathology 10: 189- 196 
Citation: Ikegami RN, Kawakami JT, Abdalla DSP, Santos RD, Filho RK, et al. (2015) Infection and Microparticles may Cause Complication of Atherosclerotic Plaques. J Diabetes Metab 6: 537. doi:10.4172/2155-6156.1000537

Page 4 of 4

8. Higuchi ML, Santos MH, Roggério A, Kawakami JT, Bezerra HG, et al. (2006) A role for archaeal organisms in development of atherosclerotic vulnerable plaques and myxoid matrices. Clinics (Sao Paulo) 61: 473-478.

9. Allen LA (2007) Phagocytosis and persistence of Helicobacter pylori. Cell Microbiol 9: 817-828.

10. Campos EG, Jesuino RS, Dantas Ada S, Brígido Mde M, Felipe MS (2005) Oxidative stress response in Paracoccidioides brasiliensis. Genet Mol Res 4: 409-429.

11. Peluso I, Morabito G, Urban L, loannone F, Serafini M (2012) Oxidative stress in atherosclerosis development: the central role of LDL and oxidative burst. Endocr Metab Immune Disord Drug Targets 12: 351-360.

12. Lu Y, Li L, Yan H, Su Q, Huang J, et al. (2013) Endothelial microparticles exert differential effects on functions of Th1 in patients with acute coronary syndrome. Int J Cardiol 168: 5396-5404.

13. Tesselaar ME, Romijn FP, van der Linden IK, Bertina RM, Osanto S (2009) Microparticle-associated tissue factor activity in cancer patients with and without thrombosis. J Thromb Haemost 7: 1421-1423.

14. Suades R, Padró T, Vilahur G, Badimon L (2012) Circulating and plateletderived microparticles in human blood enhance thrombosis on atherosclerotic plaques. Thromb Haemost 108: 1208-1219.

15. Suades R, Padró T, Alonso R, Mata P, Badimon L (2013) Lipid-lowering therapy with statins reduces microparticle shedding from endothelium, platelets and inflammatory cells. Thromb Haemost 110: 366-377.

16. Baron M, Boulanger CM, Staels B, Tailleux A (2012) Cell-derived microparticles in atherosclerosis: biomarkers and targets for pharmacological modulation? J Cell Mol Med 16: 1365-1376.

17. Duarte MI, Mariano ON, Takakura CF, Everson D, Corbett CE (1992) A fast method for processing biologic material for electron microscopic diagnosis in infectious disease. Ultrastruct Pathol 16: 475-482.
18. Homma Y (2004) Predictors of atherosclerosis. J Atheroscler Thromb 11: 265270

19. Yasim A, Ozbag D, Kilinc M, Ciralik H, Toru H, et al. (2010) Effect of atorvastatin and ezetimibe treatment on serum lipid profile and oxidative state in rats fed with a high-cholesterol diet. Am J Med Sci 339: 448-452.

20. Ravnskov U, McCully KS (2009) Review and Hypothesis: Vulnerable plaque formation from obstruction of Vasavasorum by homocysteinylated and oxidized lipoprotein aggregates complexed with microbial remnants and LDL autoantibodies. Ann Clin Lab Sci 39: 3-16.

21. Momiyama Y, Ohmori R, Taniguchi H, Nakamura H, Ohsuzu F (2004) Association of Mycoplasma pneumoniae infection with coronary artery disease and its interaction with chlamydial infection. Atherosclerosis 176: 139-144.

22. Kockx MM (1998) Apoptosis in the atherosclerotic plaque: quantitative and qualitative aspects. Arterioscler Thromb Vasc Biol 18: 1519-1522.

23. Mallat Z, Hugel B, Ohan J, Leseche G, Freyssinet JM, et al. (1999) Shed membrane microparticles with procoagulant potential in human atherosclerotic plaques: a role for apoptosis in plaque thrombogenicity. Circulation 99: 348353.

24. Leroyer AS, Isobe H, Leseche G, Castier Y, Wassef M, et al. (2007) Cellular origins and thrombogenic activity of microparticles isolated from human atherosclerotic plaques. J Am CollCardiol 49: 772-777.

25. Tesselaar ME, Romijn FP, van der Linden IK, Bertina RM, Osanto S (2009) Microparticle-associated tissue factor activity in cancer patients with and without thrombosis. J Thromb Haemost 7: 1421-1423.

26. Higuchi ML, Pierri H, Sesso A, Santos MHH, Timenestsky J, et al. (2004) Complexes of Mycoplasma pneumoniae and $\mathrm{C}$ reactive protein in elderly atherosclerotic sera. Immunology 88: 89-93. 